\title{
Editorial
}

\section{Nanomaterials for Cancer Phototheranostics}

\author{
Peng Huang, ${ }^{1}$ Daishun Ling, ${ }^{2}$ Jibin Song, ${ }^{3}$ Gang Liu, ${ }^{4}$ and Jin Xie ${ }^{5}$ \\ ${ }^{1}$ Guangdong Key Laboratory for Biomedical Measurements and Ultrasound Imaging, School of Biomedical Engineering, \\ Shenzhen University, Shenzhen 518060, China \\ ${ }^{2}$ Institute of Pharmaceutics, College of Pharmaceutical Sciences, Zhejiang University, Hangzhou 310058, China \\ ${ }^{3}$ Laboratory of Molecular Imaging and Nanomedicine, National Institute of Biomedical Imaging and Bioengineering (NIBIB), \\ National Institutes of Health (NIH), Bethesda, MD 20892, USA \\ ${ }^{4}$ Center for Molecular Imaging and Translational Medicine School of Public Health Xiamen University, Xiamen 361102, China \\ ${ }^{5}$ Department of Chemistry, Bio-Imaging Research Center, The University of Georgia, Athens, Georgia 30602, USA \\ Correspondence should be addressed to Peng Huang; peng.huang@szu.edu.cn
}

Received 5 December 2016; Accepted 7 December 2016

Copyright @ 2016 Peng Huang et al. This is an open access article distributed under the Creative Commons Attribution License, which permits unrestricted use, distribution, and reproduction in any medium, provided the original work is properly cited.

The rapid development of advanced nanotechnology promises the integration of multiple diagnostic/therapeutic modalities into one nanoplatform for cancer theranostics. This issue compiles 3 review articles and 7 high-quality original research articles related to the field of nanomaterialbased cancer theranostics.

Phototherapies, such as photothermal therapy (PTT), photodynamic therapy (PDT), or photo-triggered drug/gene delivery, have gained considerable attention because of specific spatiotemporal selectivity and minimal invasiveness. Considering the inherent biocompatibility and biodegradability of proteins and peptides, P. Huang and coworkers summarized recent advances in the development of protein/peptide-based photothermal cancer theranostics, using protein/peptide as delivery vehicles or synthesis biotemplates of PTT agents. M. G. O'Toole and coworkers developed a near-infrared (NIR) responsive oligonucleotidecoated (AS1411, hairpin, or both) gold nanoplate loaded with doxorubicin (DOX), which is demonstrated to be nontoxic to cells without triggered release, while being acutely toxic to cells after 5 minutes of laser exposure to trigger DOX release. K. Na and coworkers described an acidic tumor $\mathrm{pH}$-responsive nanophotomedicine ( $\mathrm{pH}-\mathrm{NanoPM})$, which was prepared by self-assembly of a $\mathrm{pH}$-responsive polymeric photosensitizer ( $\mathrm{pH}-\mathrm{PPS}$ ) consisting of $\mathrm{pH}-$ cleavable methoxypolyethylene glycol ( $\mathrm{pH}-\mathrm{C}-\mathrm{mPEG}$ ), for targeted PDT.
To obtain higher therapeutic efficiency, the innovative combination of phototherapies with other conventional treatments might reduce the unacceptable normal tissue toxicity while maintaining the desired tumor suppression effect. Q. Wei and coworkers reviewed the innovative combination therapy of radiotherapy (RT) and PDT, which is utilized to overcome the weaknesses of the conventional RT and PDT treatment regimen. L. Zhang and coworkers provided insights into the four different cancer treatments such as PTT, gold nanoparticle-aided PDT, gold nanoparticle-aided RT, and as drug carrier. Z. Li and coworkers developed indocyanine green conjugated silica-coated gold nanorods for simultaneously enhancing fluorescence imaging and twophoton luminescence imaging and synergistic PDT/PTT phototherapies.

For diagnosis, B. Liu and coworkers prepared graphene quantum dots with enhanced fluorescence quantum yield through the solvothermal route, which exhibited low cytotoxicity and satisfactory cell imaging performance on oral squamous cells. J. Lin, D. Cui, and coworkers developed 3D ensemble of graphene oxide (GO) and gold nanorods (GNRs) for surface-enhanced Raman scattering (SERS). The GO/GNRs substrates exhibited ultrahigh SERS activity and sensitivity of 2-mercaptopyridine (2-Mpy) with the detection limit as low as $\sim 1 \mathrm{M}$. Moreover, L. Guang-Ming, T. ZhaoGang, and coworkers conjugated NIR fluorescent heptamethine dye (IR-808) and magnetic resonance (MR) contrast 
agent (Gd-DTPA) with highly aminated mesoporous silica nanoparticles for dual-modal MR and NIR fluorescence imaging.

Besides those, Z. Zhang and coworkers investigated the ability of transfersomal gel carrying the antiscarring agent (5fluorouracil) in dermal tumor to permeate hypertrophic scars both in vivo and in vitro, using fluorescence imaging.

By compiling these papers, we hope to enrich our readers and researchers with respect to nanomaterial-based cancer phototheranostics.

Peng Huang

Daishun Ling

Jibin Song

Gang Liu

Jin Xie 

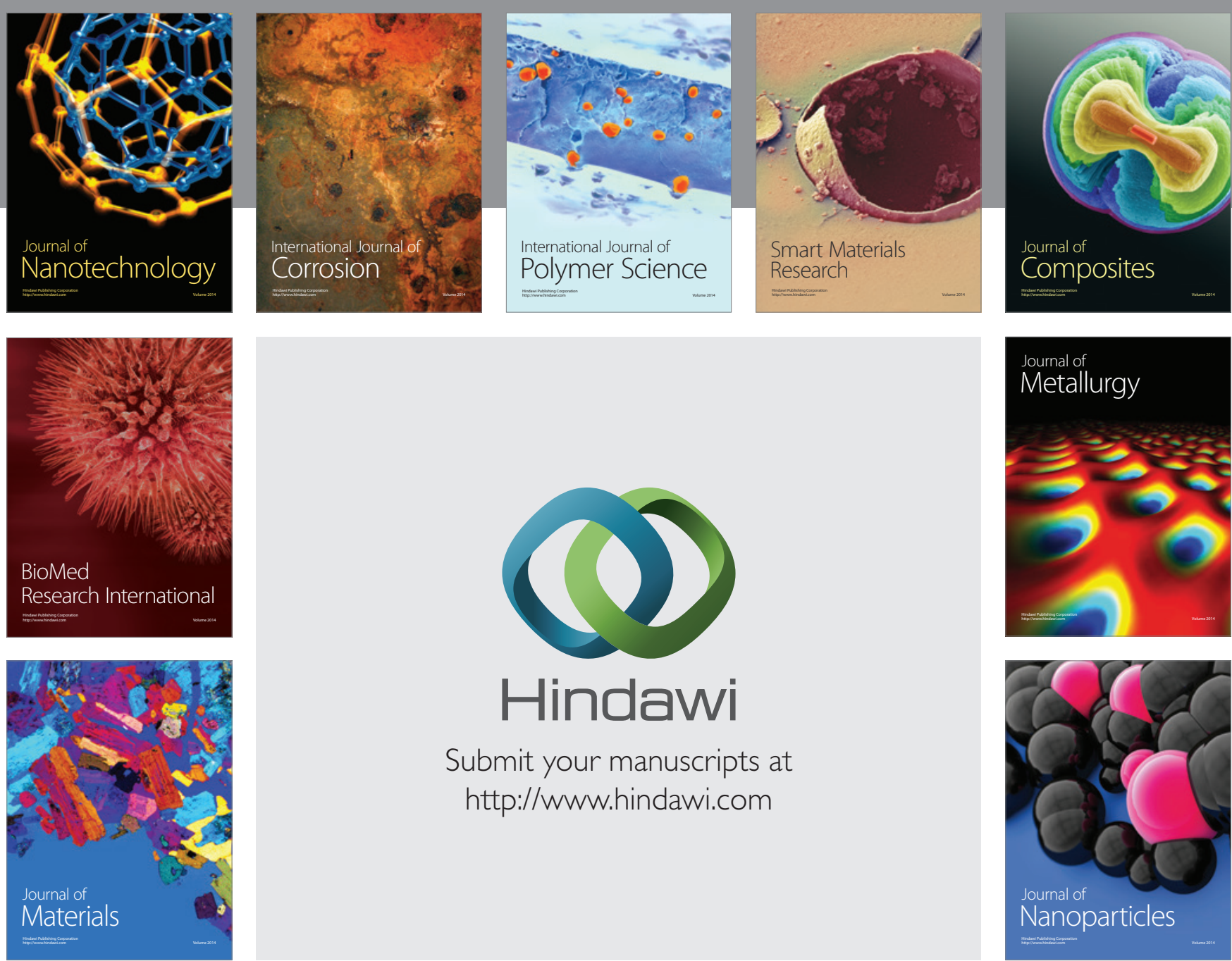

\section{Hindawi}

Submit your manuscripts at

http://www.hindawi.com

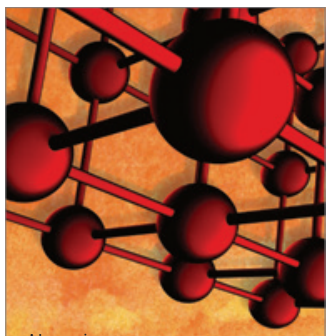

Materials Science and Engineering
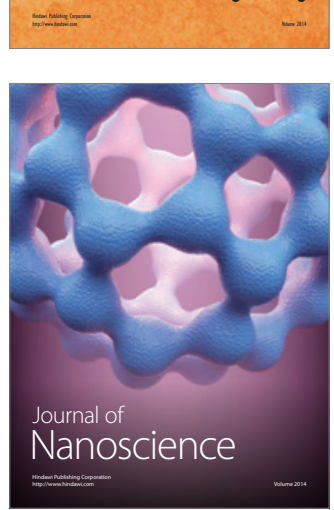
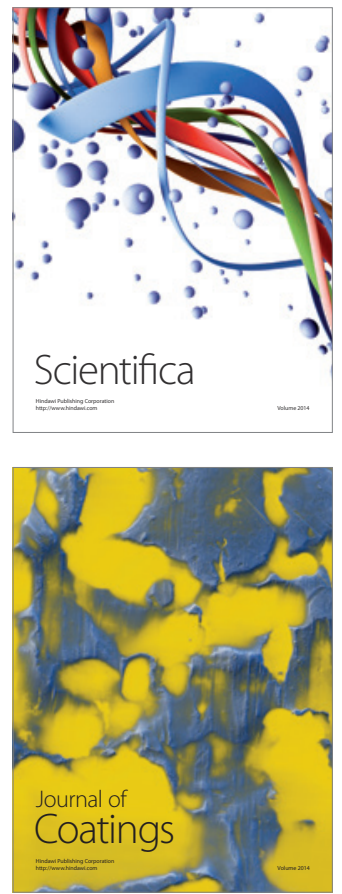
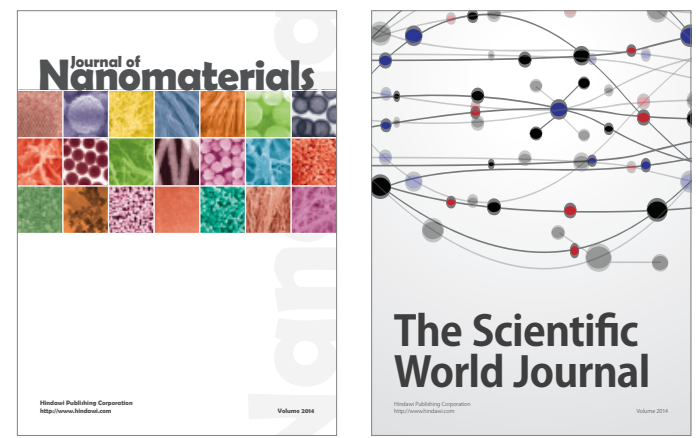

The Scientific World Journal
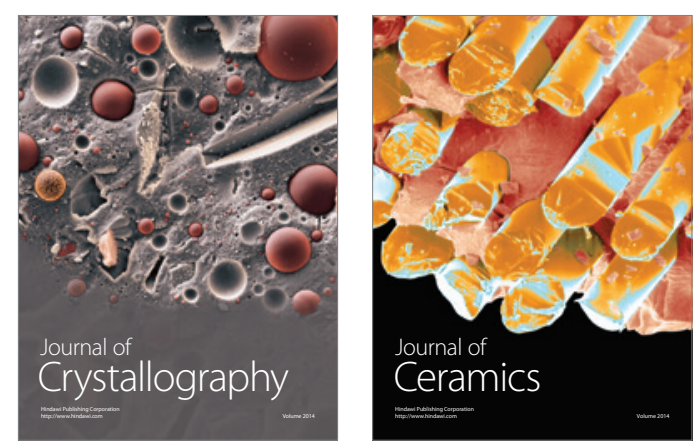
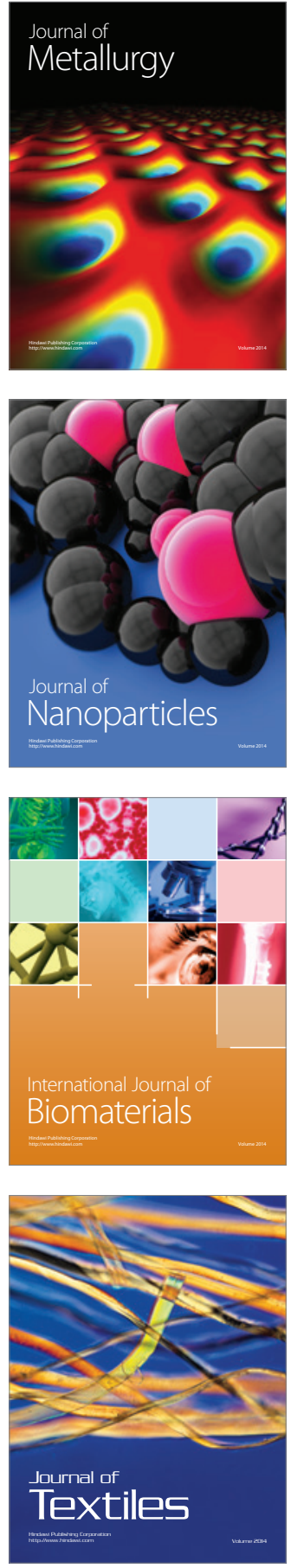